\title{
Ring like Enhancement in Dysembryoplastic Neuroepithelial Tumour (DNET)
}

\author{
Simar Abheet Singh ${ }^{1}$, Puneet Mittal ${ }^{2}$, Ranjana Gupta ${ }^{3}$, Narender Yadav ${ }^{4}$, Balkaran Singh ${ }^{5}$
}

\begin{abstract}
${ }^{1}$ Department of Radiodiagnosis, Maharishi Markandeshwar Institute of Medical Sciences and Research (MM
Deemed to be University), Mullana, Ambala, Haryana, India. ${ }^{2}$ Department of Radiodiagnosis, Maharishi

Markandeshwar Institute of Medical Sciences and Research (MM Deemed to be University), Mullana, Ambala, Haryana, India. ${ }^{3}$ Department of Radiodiagnosis, Maharishi Markandeshwar Institute of Medical Sciences and Research (MM Deemed to be University), Mullana, Ambala, Haryana, India. ${ }^{4}$ Department of Radiodiagnosis, Maharishi Markandeshwar Institute of Medical Sciences and Research (MM Deemed to be University), Mullana, Ambala, Haryana, India. ${ }^{5}$ Department of Radiodiagnosis, Maharishi Markandeshwar Institute of Medical Sciences and Research (MM Deemed to be University), Mullana, Ambala, Haryana, India.
\end{abstract}

\section{INTRODUCTION}

DNET is a low grade cortical neoplasm which closely resembles cortical dysplasia on magnetic resonance imaging (MRI). It is usually non-enhancing but can show some enhancement on about one third of case. Rarely enhancement can be ring like and when the mass is large in size, it can cause considerable diagnostic problem from higher grade gliomas. However, age of the patient and associated features like calcification, lack of mass effect and long duration of symptoms should assist in reaching the correct diagnosis. Dysembryoplastic neuroepithelial tumour (DNET) is low grade cortical glioma which is most commonly seen in temporal lobe. It is usually a non-enhancing tumour but sometimes it can show enhancement and occasionally it can be ring like, which can cause diagnostic confusion with higher grade gliomas. We report a case of DNET which showed ring like enhancement of post contrast images.

\section{PRESENTATION OF CASE}

An 11-year-old boy presented with chief complaint of recurrent episodes of generalised tonic clonic seizures from last 7 years for which he was on anti-epileptic treatment. There was no associated history of fever, trauma or developmental delay. On physical examination, patient was conscious and well oriented. There was no focal neurological deficit. MRI brain with contrast was done. It showed a heterogeneous mass lesion in left temporo-occipital region. It appeared predominantly hypointense on T1W images (figure 1A) with few hyperintense areas at the periphery. It appeared heterogeneously hyperintense on T2W (figure 1B) and FLAIR (figure 1C) images with some cystic areas. On gradient echo (GRE) images, large area of blooming was seen within the lesion. There was no significant mass effect or perilesional oedema. There was smooth scalloping of inner table of skull (arrow in 1B) indication slowly growing lesion. On post contrast images, it showed multi-focal ring like enhancement. In view of long history of intractable seizures and no mass effect of the lesion, differential diagnoses included low grade gliomas like ganglioglioma, oligodendroglioma and atypical DNET. The lesion was excised. Large areas of calcification were removed which were seen to cause blooming on GRE images. Subsequent histopathology confirmed the diagnosis of DNET.

\author{
Corresponding Author: \\ Narender Yadav, \\ Department of Radiodiagnosis, \\ Maharishi Markandeshwer Institute of \\ Medical Sciences and Research, \\ Mullana, Ambala, Haryana, India \\ E-mail: ynarender07@gmail.com
}

DOI: $10.14260 /$ jemds/2020/68

Financial or Other Competing Interests: None.

How to Cite This Article:

Singh SA, Mittal P, Gupta R, et al. Ring like enhancement in dysembryoplastic neuroepithelial tumour (DNET). J. Evolution Med. Dent. Sci. 2020;9(05):304305, DOI: $10.14260 /$ jemds/2020/68

Submission 28-11-2019,

Peer Review 09-01-2020,

Acceptance 16-01-2020,

Published 03-02-2020. 

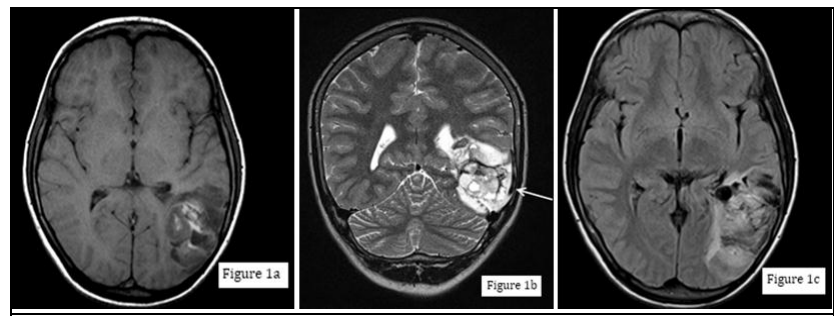

Figure 1. Axial T1W (1a) image showing focal mass lesion in left temporo-parietal region appearing predominantly hypointense with few hyperintense areas at the periphery. Coronal T2W (1b) and axial flair (1c) image showing the mass to be heterogeneously hyperintense with some cystic areas with multiple T2W hypointense areas. There is no significant mass effect or perilesional oedema.

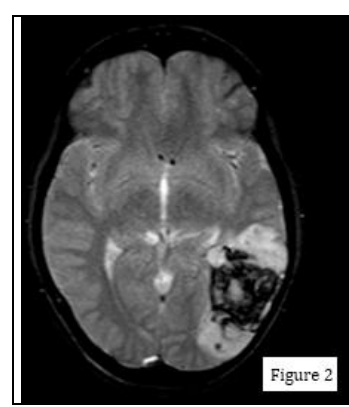

Figure 2.

Axial (2) GRE Images Showing

Extensive Areas of

Blooming within the Mass $-\mathrm{s} / \mathrm{o}$ Calcifications

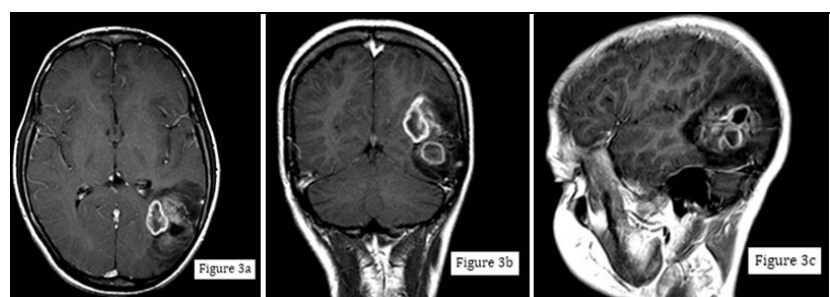

Figure 3. Axial (3a), Coronal (3b) and Sagittal (3c) Post Gad Images Showing Multi-Focal Ring Like Enhancement within the Mass

\section{DISCUSSION}

Magnetic resonance imaging (MRI) is the modality of first choice for imaging of epileptic patients. It can reliably detect hippocampal sclerosis which is the most common cause of refractory/intractable epilepsy. Second most common cause detected on MR is benign tumours like ganglioglioma, DNET and oligodendroglioma. [1] DNET is a benign cortical based tumor of neuroepithelial origin. It usually presents with refractory seizures in patients less than 20 years of age with male preponderance of cases. [2] Temporal lobe is the most common location followed by frontal lobe. [2]

On CT, they are seen as hypodense masses which characteristically cause scalloping of the inner table of skull indicating chronic slow growing lesion. [3, 4] Calcification is seen in $20-30 \%$ of cases. [4] MRI is the imaging modality of choice for imaging of these tumors and allows much more detailed evaluation. They appear predominantly hypointense on T1W and markedly hyperintense on T2W images, sometimes giving multicystic appearance. ${ }^{[3,4]}$ Usually there is no surrounding edema or mass effect.[3] Calcification can be detected by susceptibility weighted imaging. MR may also reveal scalloping of inner table of skull as is seen in our case. DNET are usually non enhancing tumors but may show some enhancement in about one third of the cases. ${ }^{[2,4]}$ However ring like enhancement pattern is very uncommon ${ }^{[4]}$ as is seen in our case. This can cause diagnostic confusion and in such cases possibility of high grade glioma must be considered. [4] However age of the patient, scalloping of inner table of skull (indicating a slowly growing tumor), absence of edema and mass effect are useful in the differential diagnosis.

\section{REFERENCES}

[1] Ostertun B, Wolf HK, Campos MG, et al. Dysembryoplastic neuroepithelial tumors: MR and CT evaluation. AJNR Am J Neuroradiol 1996;17(3):419-30.

[2] Koeller KK, Henry JM. From the archives of the AFIP: superficial gliomas: radiologic-pathologic correlation. Armed Forces Institute of Pathology. Radiographics 2001;21(6):1533-56.

[3] Gaikwad SB, Garg A, Deol PS, et al. Dysembryoplastic neuroepithelial tumors neuroimaging findings. Rivista di Neuroradiologia 2003;16:877-82.

[4] Sharma MC, Jain D, Gupta A, et al. Dysembryoplastic neuroepithelial tumor: a clinicopathological study of 32 cases. Neurosurg Rev 2009;32(2):161-9, discussion 16970. 